INTERNATIONAL JOURN AL OF RESEARCHES IN BIOSCIENCES, AGRICULTURE AND TECHNOLOGY (c) VISHWASHANTI MULTIPURPOSE SOCIETY (Global Peace Multipumose Socie ty) R. No. MH-659/13(N) www.vmsindia.org

\title{
TAXONOMIC DIVERSITY OF PLANKTONIC ALGAE FROM JUNNAR TALUKA OF PUNE DISTRICT (INDIA)
}

\author{
Radhakishn N. Tagad \\ Department of Botany, Hon. B. J. College, Ale, Tal. Junnar, Dist. Pune. \\ tagadrn@rediffmail.com, tagadrn@gmail.com
}

\begin{abstract}
:
The Junnar tehsil in Pune District is situated between 19011'59" Northern 73052'47" Eastern latitude on the northern part of Deccan Plateau \& composed of undulating hills. Junnar tehsil is famous for its wells and Dams. The famous and historical fort of Shivne ri where Shivaii Maharai was born is in this region. There is also a Satellite Center in Arvi. Survey was done of the following impoundments - Manikdoha Dam, Yedgaon Dam, Pimpalgaon joge Dam, and Wadaj Dam. Sub-aerial algae growing attached to tree barks, on damp walls or other such substrata were collected by scraping with a scalpel and then picked up with the help of a forceps. All algal plants were identifie d up to genus, species, variety as well as formas level. The 344 species, 99 varieties, 10 forma, 2 Variety and forma belonging to 112 genera of 39 familes from 12 orders of 06 classes from 5 divisions. Family Des midiaceae includes 10 genera, 38 species and 9 varieties while family Hyellaceae, Ceratiaceae, Chaetoceraceae, Chlorochytriaceae, Treubariaceae, Haematococcaceae and Chlorococcaceae include only 1 genus and 1 species; Cosmarium is more densely occur in Ambegaon tehsil.
\end{abstract}

Keywords: Pune, Junnar, Planktons, Cosmarium.

\section{Introduction:}

Filamentous algae were collected from mass growths by hand. Sub-aerial algae growing attached to tree barks, on damp walls or other such substrata were collected by scraping with a scalpel and then picked up with the help of a forceps. Hand collected samples were investigated from April 2014 to October 2015. The present investigation is undertaken with keep in mind that to study the algal population from selected stations of study area.

Attempts have been undertaken to bring out the Algal flora of this region. 37 Nostocales are described in the present paper. In which family Oscillatoriaceae cons is ts genus Lyngbya \& Oscillatoria in which genus Lyngbya consists 10 species, Oscillatoria consists 21 species; while family Scytonemataceae consists genus Scytonema that consists 6 species.

\section{Materials and Methods:}

The samples were preserved in a mixture of $50 \mathrm{ml}$ of $95 \%$ ethyl alcohol, $5 \mathrm{ml}$ of glacial acetic acid, $10 \mathrm{ml}$ of $40 \%$ commercial formalin and $35 \mathrm{ml}$ of water. The specimens are observed under microscope for 10X, 40X, 100X and Photographs were taken with the help digital camera under appropriate magnifications. Identification of specimens was mostly based on the keys given in standard monographs \& literatures. The Vauc her specimens have been deposited at Dept. of Botany, Hon. Baladaheb Jadhav College, Ale, Tal. Junnar, Dist. Pune.

Periodical collections of algae from the study area we re done from the Dams as well as Rivers, Lake's, Puddles, Pulls etc. from Junnar
Tehsil. Sampling stations were carried away. The samples were bringing to laboratory for identification; Identification were done with the help of Indian monographs and other standard literature like Desikachari (1959), Randhawa (1959), Venkatraman (1961), Prescott (1951), Ramnathan (1964), Bourrlly (1970), Philipose (1967), Gonzalvies (1981), Iyengar and Desikachari (1981), Desikachari et al (1990), Anand (1998) and Sarode and Kamat (1984). The collected algal forms had been preserved in $4 \%$ formalin.

\section{List of Planktonic Algal specimens} collected from Study Area:

Division:Chlorophyta $>$ Class:Chlorophyceae $>$ Order: Volvocales>F amily: Volvocaceae.

1. Volvox aureus Ehrenberg

2. Volvox globator Linnem. Ehrb.

3. Gonium pectorale Mulle $\mathrm{r}$

4. Gonium sociale (Duj.) Warming in Bot.

5. Pandorina morum (Muller) Bory

6. Eudorina elegans Ehrenberg.

Family: Chlamydomonadaceae

1. Chlamydomonas globosa Snow

2. Chlamydomonas polypyrenoideum Prescott

3. Chlamydomonas sphagnicola Fritsch and Takeda

4. Sphaerellopsis iyengarii Balakrishnan

Family: Haematococcaceae

1. Haematococcus lacustris (Girod) Ros tafinski

Order: Tetrasporales>Family: Palmellaceae

1. Gloeocystis ampla (Kuetz) Lagerheim

2. Sphaerocystis schroeteri Chodat. 
Order:

Clorococcales>Family:

Clorococcaceae

1. Chlorococcum humicola (Naeg.) Rabenhorst

Fa mily: Chlorochytriaceae

1. Kentrosphaera bristolae G. M. Smith

Family: Micractiniaceae

1. Golenkinia radiata (Chod.) Wille

2. Micractinum pusillum Fresenius

3. Micractinum quadrisetum (Lemm.) G.M. Smith

4. Errerella bornhemensis Conrad.

5. Trochiscia aciculifera (Lagerh.) Hans girg

Family - Dictyosphaeriaceae

1. Dictyospherium pulchellum Wood

2. Westella linearis G. M. Smith

3. Schroederia indica sp. nov.

4. Schroederia judayi G. M. Smith

Fa mily: Hydrodicty aceae

1. Pediastrum boryanum (Turpin) Menegbini

2. Pediastrum duplex Meyen v. clathratum (A. Braun) Lagerheim

3. Pediastrum duplex Meyen v. gracillimum West and We st

4. Pediastrum duplex Meyen v. reticulatum Lagcrh.

5. Pediastrum integrum Naegeli

6. Pediastrum integrum Naegeli v. perphoratum Raciborski

7. Pediastrum ovatum (Ehr.) A. Braun

8. Pediastrum simplex (Meyen) Lemme rmann

9. Pediastrum simplex (Meyen) Lemme rmann v. duodenarium (Bailey) Rabenhorst

10. Pediastrum tetras (Ehre nb.) Ralfs

11. Pediastrum tetras (Ehrenb.) Ralfs. v. apiculatum Fritseh

12. Sorastrum spinulosum Naegeli

13. Tetraedron caudatum (Corda) Hansgirg

14. Tetraedron gracile (Reinsch) Hansgirg

15. Tetraedron minimum (A. Braun) Hans girg

16. Tetraedron regulare Kuetzing $\mathrm{v}$. bifarcatum Wille

17. Tetraedron regulare Kuetzing v. incus Teiling f. major Prescott

18. Tetraedran trigonum (Naegali) Hansg.

19. Tetraedron trigonum (Naegali) Hans g. v. longispinum var. nov.

Family: Coelastrace ae

1. Coelastrum cambricum Archer

2. Coelastrum cambricum Archer v. intermedium Bohlin G.S. West

3. Coelastrum microporumNaegeli

4. Coelastrum proboscideum Boh lin

5. Coelastrum sphaericum Naegeli
Family: Selenastraceae

1. Selenastrum bibraianum Reinsch

2. Selenastrum gracile Reinsch

3. Selenastrum minutum (Naegeli) Collins.

4. Selenastrum westii G. M. Smith

5. Kirchneriella lunaris (Kirch) Moebius

6. Kirchneriella lunaris (Kirchner) Moebius v. dianae Bohlin

7. Kirchneriella lunaris (Kirchner) Moebius v. irregu laris G.M. Smith

8. Kirchneriella obesa (W.West) Schmidle v. a perta (Teil.) Brunnthaler

9. Ankistrodesmus convolutus Corda

10. Ankistrodesmus falcatus (Corda.) Ralfs. v. mirabilis (West and West)

11. Ankistrodesmus falcatus (Corda.) Ralfs. v. radiatus (Chodat) Lemme.

12. Ankistrodesmus falcatus (Corda.) Ralfs. v. sp irilliformis (W. et West) G. S.West

13. Ankistrodesmus fractus (West and West) Brunnthaler

14. Ankistrodesmus spairalis (Turner) Lemme rmann

15. Ankistrodesmus spiralis(Turner) Lemm. v. faciculatus G.M. Smith

16. Quadrigula lacustris (Chod.) G.M. Smith

17. Actin astrum gracilimum G.M. Smith

18. Actinastrum hantzschii Lage rhe im

19. Actinastrum hantzschii Lage rheim v. fluviatile Schroeder.

Family: Scenedesmaceae

1. Scenedesmus abund ans (Kirch.) Chodat

2. Scenedesmus acuminatus (Lagerheim) Chod at f. globosus Horto b. et Neme th

3. Scenedesmus acuminatus (Lagerh.) Chod. v. bernardii (G. M. Smith) Deduss

4. Scenedesmus acutus Meyen

5. Scenedesmus armatus v. bicaudatus (Guglie lme tti) Ghodat

6. Scenedesmus bernard ii G. M. Smith

7. Scenedesmus bijugatus (Turp.) Lagerheim

8. Scenedesmus bijugatus(Turpin) Kuetzing f. irregularis (Wille)

9. Scenedesmus bijugatus (Turpin) Kuetzing. v. graevenitzii (Be rnard) comb. Nov

10. Scenedesmus carinatus (Lemm.) Chod.

11. Scenedesmus denticulatus Lagerheim

12. Scenedesmus incrassatulus Bohlin

13. Scenedesmus intermedius Chod. $v$. bicaudatus Horto b.

14. Scenedesmus longispina Chod.

15. Scenedesmus longispina Chod. v. capricornus Skuja Horto b n.f .

16. Scenedesmus obliquus (Turpin) Kuetzing

17. Scenedesmus opoliensis $\mathrm{P}$. Richter

18. Scenedesmus perforatus Lemmermann

19. Scenedesmus quadricauda (Turp.) Breb.

20. Tetrallantos lagerheimii Telling 
21. Scenedesmus carinatus (Le mm.) Chod.

22. Scenedesmus denticulatus Lagerheim

23. Scenedesmus incrassatulus Bohlin

24. Scenedesmus intermedius Chod. $v$. bicaudatus Horto b.

25. Scenedesmus long ispina Chod.

26. Scenedesmus long ispina Chod. v. capricornus Skuja Hortob n.f .

27. Scenedesmus obliquus (Turpin) Kuetzing

28. Scenedesmus opoliensis $\mathrm{P}$. Richter

29. Scenedesmus perforatus Lemmermann

30. Scenedesmus quadricauda (Turp.) Breb.

31. Crucigenia apiculata (Lemm.) Schmidle

32. Crucigenia irregularis Wille.

33. Crucigenia lauterbornii Schmidle

34. Crucigenia quadrata Morre $n$

35. Crucigenia rectangularis (A. Braun) Gay

36. Crucigenia truncata G. M. Smith

37. Tetrastrum staurogeniaeforme (Schroeder) Le mmermann

Family: Treubariaceae

Treubaria triappend iculata Be rnard

Family: Oocystaceae

1. Chlorella conclomerata (Artari) Oltmanns

2. Chlorella vulgaris Beyerinck

3. Oocystis elliptica W.West

4. Oocystis gloeocystiformis Borge

5. Oocystis kumaonensis K. P. Singh

6. Oocystis lacustris Chod at

7. Oocystis macrospora (Turner) Brunnthaler

8. Oocystis nodulosa We st and West

9. Oocystis novaese mliae Wille

10. Oocystis pusilla Hans girg

11. Oocystis solitaria Wittrock

12. Oocystis submarina Lagerheim

13. Lagerhe imia subsalsa Lemmermann

14. Nephrocytium obesum W. et G. S. West.

Family: Botryococcaceae

1. Botryococcus braunii Kuetzing

2. Geminella ordinata (W.et G.S. West) Heering.

Order:

Cladophorales>Fa mily:

Cladophoraceae

1. Cladophora fracta (Dillw.) Kuetzing v. lacustris (Kuetz.) Brand ex Heering

2. Pithophora varia Wille

3. Rhizoclonium crassipellitum West and West

4. Rhizocloniu m hookeri Kuetzing.

Order: Zygnematels>Family: Zygnemataceae

1. Mougotia americana Transeau

2. Mougotia caimani Transeau

3. Mougotia floridana Transeau

4. Mougotia gotlandica (Cleve) Wittrock
5. Mougotia regellii Skuja

6. Mougotia sphaerocarpa Wolle

7. Mougotia tenuissima (de Bary) Czurda

8. Debarya smithii Transeau

9. Zygnema czurdae Randhawa

10. Zygnemopsis decussata Transeau

11. Zygnemopsis lamellata Randhawa

12. Sirogonium tenuis (Nordstedt) Transeau

13. Temnogametum indicum Iyengar

14. Temnogametum uleanum (Mobius) Wille

Family: Mesotaeniaceae

1. Mesotaenium giganteum Turner

2. Netrium digitus (Ehr.) Itzigs. et Rothe

3. Cylindrocystis brebissonii Menegh.

Family: Desmidia ceae

1. Staurodesmus pterosporum (Lundell) comb. nov.

2. Staurastrum gracile v. elegantulum West et West

3. Staurastrum leptocladum v. cornutum Wille

4. Staurastrum margaritaceum (Ehrenb.) Me neghini

5. Staurastrum panamanum sp. Nov.

6. Staurastrum punctulatum B reb.

7. Staurastrum sebaldi Reinsch. v. gracile Messik

8. Staurastrum sebaldi Reins ch.v. ornatum Nordst.

9. Staurastrum sparsidentatum Nygaard

10. Staurastrum tetracerum Ralfs

11. Closterium acerosum (Schrank) Ehre nberg

12. Closterium dianae Eh renberg

13. Closterium intermedium Ralfs.

14. Closterium lanceolatum Kue tzing

15. Closterium leibleinii Kue tz.

16. Closterium moniliferum (Bory) Ehre nberg

17. Closterium ven us Kuetz.

18. Pleurotaenium ehernbergii (Brebisson) DeBary

19. Euastrum ansatumEhren berg

20. Euastrum irregu lar Gonzalves and Gangle v. gwaliorensi v. nov.

21. Euastrum oblongum (Greville.) Ralfs

22. Micrasterias americana (Ehrenbe rg) Ralfs.

23. Micrastrias apiculata (Ehrenberg) Me neghini

24. Cosmarium awadhense Prasad and Mehrotra

25. Cosmarium biretum Brebisson v. trigiberrum No rd stedt.

26. Cosmarium botrytis Me neghini

27. Cosmarium contractum Kirchn.

28. Cosmarium contractum v. ellipsoidium (Elfv.) West et West

29. Cosmerium cucu mis (Corda) Ralfs

30. Cosmarium depressum (Naegeli) Lundell

31. Cosmarium holmiense Lundell. v. integrum Lundell 
32. Cosmarium meneghinii $\mathrm{B}$ rebisso $\mathrm{n}$

33. Cosmarium miscellum Skuja

34. Cosmarium moniliforme (Turp.) Ralfs

35. Cosmarium nitidulum De Notaris

36. Cosmarium pachdermum Lundell v. aethiopicum W. and G.S. West

37. Cosmarium portianum Archer

38. Cosmarium quadrum Lundell.

39. Cosmarium quinarium Lundell

40. Cosmarium retusiforme(Wille) Gutw

41. Cosmarium sexnotatum Gutw.

42. Cosmarium subcrenatum Hantzsch.

43. Cosmerium subimpressulum Borge.

44. Arthrodesmus convergens Ehrenberg

45. Arthrodesmus phimus Turner

46. Spondylosum planum (Wolle) W. and G.S. West.

47. Hyalothca dissiliens (Smith) Brebisson

Division: Euglenophy ta>Class:

Eugle nophyceae>Order: Euglenales $>$ Family : Euglenaceae

1. Euglena acus Ehrenberg

2. Euglena acus Ehrenberg v. rigida Huebner

3. Euglena geniculata Dujard in

4. Euglena gracil is Kle bs.

5. Euglena limnophila Lemm.

6. Euglena minuta Pre scott

7. Euglena oblonga Schmitz

8. Euglena oxyuris Schmarda

9. Euglena polymorpha Dangeard.

10. Euglena proxima Dangeard

11. Euglena rostrifera L.P. Josnson

12. Euglena sangu inea Ehrenberg

13. Euglena satelles Brast. Spect

14. Euglena spiroides Lemmermann

15. Euglena tuba H. J. Carter

16. Lepocinclis fusiformis (Carte $r$ ) Lemmermann

17. Lepocenalis texta (Duj.) Lemm

18. Phacus curvicauda Swire nko

19. Phacus helicoides Pochmann

20. Phacus lismorensis Playfiar

21. Phacus longicauda (Ehrenb.) Dujard in

22. Phacus longicauda (Ehr.)Dur.v. insecta Hub Pestaio.

23. Phacus pulcher Roll.

24. Phacus ranula Pochm

25. Phacus swirenkoi Skvortzow

26. Phacus tortus (Lemm) Skvortzow

27. Trarhelomonas dubia (Swir.)Defland re

28. Trarhelomonas intermedia Dangeard

29. Trachelomonas rotundaSwirenko

30. Trachelomonas volvocina Ehr.v. punctate Playfair

Division:Chrysophyta $>$ Class :Chrysophycea e> Order:Chrysomonadales>

Family:Mallomonadaceae.

1. Mallomonas acaroarides $\mathrm{v}$. maskovensis (We rmel) Krieger

2. Mallomonas tonsurata Teiling
Class: Ba scillariophyceae $>$ Order: Centrales $>$ Suborder: Coscinodiscineae $>$ Family:

Coscinodiaceae> Subfa mily: Melosiroideae

1. Melosira granulata (Ehr.) Ralfs v. angustissima $\mathrm{O}$. Muell.

2. Melosira islandica O. Muell

3. Melosira juergensii Agardh

Subfamily: Coscinodiscoideae

1. Cyclotella meneghiniana Kuetz.

2. Cyclotella striata (Kuetz.) Grun.

Suborder:Biddulphineae>

Family:Chaetoceraceae

1. Attheya zachariasi J. Brun

Order: Pinnales $>$ Suborder:

Fragilarineae $>$ Family: Fragilariaceae

1. Fragilaria brevistriata Grun. v. vidarbhensis $\mathrm{v}$. nov.

2. Fragilaria intermerdia Grun.

3. Fragilaria rumpens (Kuetz) Carl. v. meneghiniana (Grun) Gandhi

4. Fragilaria ungeriana Grun.

5. Synedra capitata Ehrenberg

6. Synedra ulna (Nitz.) Ehr.

7. Synedra ulna (Nitz.) Ehr. f. staurodestituta Pant.

8. Synedra ulna (Nitz.) Ehr.v. amphirhynchus (Ehr.) Grun

9. Synedra ulna (Nitz.) Ehr.v. subaequalis Grun

Family: Eunotiaceae

1. Eutonia formica A. Be rg.

2. Eunotia gracilis (Ehre nb.) Rabh.

3. Eunotia lunaris (Ehr.) Grun.

4. Eunotia rostellata Hustedt

Suborder: Achninthineae>Family :

Achnanthaceae

1. Achnanthes elata (Leud-Fort) Gandhi

2. Achnanthes inflate (Kuetz.) Grun.

3. Achnanthes inflate (Kuetz.) Grun. f. elatoides Cholnoky

4. Achnanthes lanceolate (Breb.) Grun.

5. Achnanthes trigibba Hus tedt.

Family: Naviculaceae

1. Diploneis subovalis Cleve

2. Calone is bacillum (Grun.) Me resch.v. fontinalis (Grun.) Mayer

3. Calone is beccariana Grun.

4. Calone is beccariana Grun. v. hustedtii Gandhi

5. Calone is silicula (Ehr.) Cleve v. genuinaMayer

6. Calone is silicula (Ehr.) Cleve v. minuta (Grun.)

7. Calone is silicula (Ehr.) Cleve v. truncatula (Grun.)Mayer

8. Calone is ventricosa (Ehr.) Meist. v. alpina (Cleve) Patr.

9. Gyrosigma attenuatum Motum (Kue tz.) Rabh.

10. Gyrosigma bhusavalensis sp. nov.

11. Pinnularia arraniensis Hustedt $f$. indica $\mathrm{f}$. nov. 
12. Pinnularia borealis Ehr.

13. Pinnularia borealis Ehr. v. lonavlensis Gandhi

14. Pinnularia braunii (Grun.) Cleve v. amphice phala (Mayer) Hustedt.

15. Pinnularia episcopalis Cleve

16. Pinnularia major (Kuetz.) Cleve v. linearis Cleve

17. Pinnularia major (Kuetz.) Cleve v. sendaiensis Hustedt

18. Pinnularia mesolepta Ehr. v. stauroneiformis Grun.

19. Pinnularia neglecta (Mayer) A. Berg v. undulata Gand hi

20. Pinnularia platycephala (Ehr.) Cleve

21. Pinnularia pseudoluculenta Gandhi

22. Pinnularia stauroptera (Rabh.) Cleve v. parva Grun.

23. Pinnularia stomatophorides Maye $\mathrm{r}$. ornata A. Cl. f. erlangen sis Maye $\mathrm{r}$

24. Pinnularia subcapitata Greg. v. hilseana (Jan.) O. Muell.

25. Pinnularia subcapitata Greg. v. lapponica A. Cl.

26. Navicula cari Ehr. v. angusta Grun.

27. Navicula cincta (Ehr.) Kuetz. v. heufleri (Grun.) Cleve

28. Navicula cryptocephala Kuetz. v. veneta (Kue tz) Grun.

29. Navicula cuspidata Kuetz. v. ambigua (Ehr.) Cleve

30. Navicula dice phala (Ehr.) W. Smith v. sphaerophora A. Cl.

31. Navicu la feuerborni (Feuerb.) Hustedt

32. Navicula gracil is Ehr.

33. Navicula grimii Krasske

34. Navicula mutica Kuetz. f. intermedia Hustedt.

35. Navicula pupula Kuetz v. rostrate Hustedt.

36. Navicula radiosa Kuetz. v. minutissima (Grun.) Cleve

37. Navicula similis Krasske

38. Navicula subhamulata Grun.

39. Navicula viridula Kuetz. v. capitata Mayer.

Family: Gomphone mataceae

1. Gomphonema aequa toriale Hustedt

2. Gomphonema clavetoides Gandhi v. valida Gandhi

3. Gomphonema constrictum Ehr. v. capitata (Ehr.) Cleve

4. Gomphonema constrictum Ehr. v. indica Gandhi

5. Gomphonema gracile Ehr.

6. Gomphonema gracile Ehr. f. turris Hustedt

7. Gomphonema gracile Ehr. v. auritumA. Br.

8. Gomphonema gracile Ehr. v. intricatiforme Mayer

9. Gomphonema gracile Ehr. v. lanceolate (Kuetz.) Cleve

10. Gomphonema herbidense (Greg.) Ehr.

11. Gomphonema intricatum Kuetz $v$. fossile Pant

12. Gomphonema lacus-rankala $\mathrm{G}$ andhi $\mathrm{v}$. robusta Gand hi

13. Gomphonema lancoelatum $\mathrm{Ehr}$.

14. Gomphonema monantum Schum. v. acuminatum Maye $r$

15. Gomphonema moniliforme Gandhi

16. Gomphonema olivaceum (Lyng.) Kuetz.

17. Gomphonema olivaceoides Husted $t$.

18. Gomphonema parvulum (Kue tz.) Grun.

19. Gomphonema parvulum (Kue tz.) Grun. v. micropus (Kuetz) Cle ve

20. Gomphonema sphaerophorum Ehr.

21. Gomphonema subapicatum Fritsch et Rich

22. Gomphonema subapicatum Frits ch et Rich v. okamurae (Sk'v.) Gandhi

Family: Cy mbellaceae

1. Cymbella aspera(Ehr.) Cleve

2. Cymbella bengalensisGrun.

3. Cymbella cistula (Hemp.) Grun.v. woosungensis Voigt

4. Cymbella gracilis(Rabh.) Cleve

5. Cymbella lanceolata(Ehr) V. H.

6. Cymbella perpusilla A. C1.

7. Cymbella tumida(Breb.)V. H.

8. Cymbella tu mida( Breb.) V. H. f. ventrincosa Gandhi

9. Cymbella tumidula Grun.

10. Cymbella turgida (Greg.) Cleve

11. Amphora acutiuscula Kuetz

12. Epithe mia sorex Kuetz.

13. Epithemia zebra (Ehr.) Kuetz v. frickei A. Cl.

14. Epithemia zebra (Ehr.) Kuetz v. proboscidea (Kuetz.) Grun.

15. Rhopalodia gibba( Ehr.) Muell.

Family: Nitzschiaceae

1. Hantzschia a mphioxys (Ehre nb.) Grunow. v. capitata O. Mull

2. Hantzschia a mphioxys (Ehr.) Grun. v. densestriata (Font.) A. Cl.

3. Hantzschia a mphioxys (Ehr.) Grun. v. mugadensis Gandhi

4. Nitzschia apiculata (Gorg.) Grun.

5. Nitzschia closteriumW. Smith

6. Nitzschia heufleriana Grun.

7. Nitzschia heufleriana Grun.v. elonga tea Pant.

8. Nitzschia hungarica Grun.

9. Nitzschia jugata Gandhi

10. Nitzschia obtusa W. Smith v. scalpe lliformis Grun.

11. Nitzschia palea (Kuetz.) W. Smith

12. Nitzschia theramalis kue tz.v .minor Hilse

13. Nitzschia tryblionella Hantzsch $\mathrm{v}$. levidensis (W. Smith) Grun

Family: Surirellaceae

1. Surirella apiculata W. Smith

2. Surirella biseriata Breb.

3. Surirella carponii Breg

4. Surirella ovata Kuetz 
5. Surirella ovata Kuetz.v. pinnata (W. Smith) Husted t

6. Surirellar obusta Ehr.

7. Surirella spiralis Kue tzing

8. Surirella tenera Greg. v. nervosa A. S.

9. Cymatopleura solea (Breb)

Division: Pyrrophyta $>$ Class:

Dinoph yceae> Order: Peridiniales $>$ Fa mily:

Glenodiniaceae

1. Glenodinium borgei (Lemm.) Schiller

2. Glenodinium pulvispculus (Ehre $\mathrm{nb}$ ) Stein

3. Glenodinium quadridens (Stein) Schiller

4. Peridinium cinctum (Mue ll.) Eh renberg Slein

5. Peridinium pusillum (Penard) Lemme rmann

Family: Ceratiace ae

1. Ceratium hirundinella (O.F. Muell.) Dujard in

Division: Cyanophyta>Class: Cyanophyceae> Order: Chroooccales>Family: Chroooccaceae

1. Microcystis aeruginosa Kuetz v. major (Wittr.) G. M. Smith

2. Microcystis elabens (Breb.) Kuetz.

3. Microcystis flos-aquae (Wittr.) Kirchner

4. Microcystis orrisica W. West.

5. Microsystis robusta (Clark) Nygaard

6. Microcystis viridis (A. Br.) Lemm.

7. Chrococcus cohaerens (Breb.) Nageli

8. Chroococcus limneticus Lemm.

9. Chroococcus limneticus Lemmermann v. elegans G. M. Smith

10. Chroococcus minor (Kuetz.) Naegeli

11. Chroococcus minutus (Kuetz.) Nageli.

12. Chroococcus prescottii Drouet and Daily in Droue t

13. Chroococcus tenax (Kirchn.) Hieron.

14. Chroococcus turgidus (Kutz) Naeg.

15. Chroococcus turgidus (Kuetz.) Naeg. v. maximus Nygaard

16. Chroococcus varius $\mathrm{A}$. $\mathrm{Br}$.

17. Gloeocapsa coracina Kuetz.

18. Gloeocapsa crepid inum Thuret

19. Gloeocapsa fuscolutea Nageli

20. Gloeocapsa granosa (Berk.) Kuetz.

21. Gloeocapsa punctata Nageli

22. Gloeothece linearis Nageli

23. Gleothece linear is Naegeli v. composita G. M. Smith

24. Gloeothece rupestris (Lyngb.) Bornet

25. Aphanocapsa de licatissima W. et G. S. West

26. Aphanocapsa koordersi Strom

27. Aphanocapsa pulchra (Kuetz.) Rabenh

28. Aphanocapsa rivularis (Carm.) Rabenhorst

29. Aphanothece caldaorum Richter, P.

30. Aphanothece castagnei (Breb.) Rabenhorst

31. Aphanothece clathrata W. et G. S.
West

32. Aphanothece gelatinosa (Henn.) Lemm.

33. Aphanothece nidulans Richter, $P$.

34. Aphanothece saxicola Naegeli

35. Aphanothece stagnina (Spreng.) A. Br.

36. Coelosphaerium dubium Grunow

37. Coelosphaerium kuetingianum Naegeli

38. Synechococcus elongatus Nageli

39. Synechocystis pevalekii Erce govie

40. Dactylococcopsis fascicularis Lemm. f. ind ica $\mathrm{f}$. nov.

41. Merismopedia convolutaBreb.

42. Merismopedia elegans A. Braun

43. Merismopedia elegans A. Braun v. major G. M. Smith

44. Merismopedia glauca (Ehre nb.) Naeg.

45. Merismopedia minima Beck

46. Merismopedia punctata Meyen

47. Merismopaedia tenu issima Lemm.

Family: Hyellaceae

1. Xenococcus kerneri Hansg.

Order: Nostocales>Family: Os cillatoriaceae

1. Arthrospira gomontiana Setchell

2. Spirulina gigantea Schmidle

3. Spirulina labyrinthiformis (Menegh.) Gomont

4. Spirulina major Kue tz.ex. Gomont

5. Spirulina meneghiniana Zanard. ex Gomont

6. Spirulina princeps W. et G. S. West

7. Spirulina subtilissima Kue tz ex Gomont

8. Oscillatoria agardhii Gomont

9. Oscillatoria amphigranula ta Van Goor

10. Oscillatoria chalybea (Mertens) Gomont

11. Oscillatoria chalybea (mertens) Gomont v. insularis Gardne $\mathrm{r}$

12. Oscilla toria curviceps Ag. ex Gomont

13. Oscillatoria irrigua Kuitz. Gomont

14. Oscillatoria limosa Ag. ex Gomont

15. Oscillatoria margaritifera (Kuetz) Gomont

16. Oscillatoria minnesotensis Tilde $\mathrm{n}$

17. Oscilla toria nigra Vaucher

18. Oscillatoria obscura Bruhl et Biswas

19. Oscillatoria princeps Vauche $\mathrm{r} \mathrm{ex}$ Gomont

20. Oscillatoria princeps Vauche $\mathrm{r} e \mathrm{x}$ Gomont v. pseudolimosa Ghose

21. Oscillatoria raciborskii Wolosz.

22. Oscillatoria rubescens DC ex Gomont

23. Oscillatoria salina Biswas f. major $\mathrm{f}$. nov.

24. Oscillatoria sancta (Kutz.) Gomont

25. Oscillatoria simplicissima Gomont

26. Oscillatoria vizgapatensis Rao C.B.

27. Phormidium ambigu um Gom.

28. Phormidium anomala Rao, C. B.

29. Phormidium usterii Schmidle 
30. Lyngbya baculum Gomont

31. Lyngbya digueti Gomont

32. Lyng bya hitron ymusii Lemm.

33. Lyng bya magnifica Gardner

34. Lyng bya majuscula Harvey ex Gomont

35. Lyng bya subconfervoides Borge

36. Lyng bya versicolor (Wartmann) Gomont

37. Microcoleus lacu stris (Rabenh.) Farlow.

Family: Nostocaceae

1. Anabaenopsis arnoldii Aptekar

2. Cylindrospermum muscicola Kutzing ex Born.et Flah. v. kashmiriensis Bharadwaja

3. Anabaena ambigua Rao C. B.

4. Anabaena ballyganglii Bane rji

5. Anabaena khannae Skuja

6. Anabaena laxa (Rabehn.)

7. Anabaena naviculoides Frits $\mathrm{ch}$

8. Anabaena oscillarioides Bory ex Bron. et Flah.

9. Anabaena variabilis Kuetz. ex. Born. et Flah.

10.Nostoc carnaeumAg.ex. Born. et Flah.

11.Nodularia spumigena Me rtens ex Born.et Flah.

Family: Scytonemataceae

1. Plectonema tomasinianum (Kutz.) Born. ex. Gomont

2. Scytonema chiastum Geitler

3. Scytone ma crustaceum Ag. ex Born. et Flah.

4. Scytone ma geitleri Bharadwaja

5. Scytonema mirabile (Dillw.) Born

6. Scytonema pseudopunctatum Skuja

Family: Rivulariaceae

1. Gloeotrichia kurziana Zeller orth. Mut.

2. Gloeotrichia raciborskii Woloszynska v. conica Dixit.

3. Homoeothrix hansgirgi (Schmidle) Lemmermann.

\section{References:}

Anand, N. and G. Revathi; 'Blue-Green Algae from Rice Fields of Tamil Nadu'; Phykos 26:17$21: 1987$

Anand, N.; 'Indian Fresh Water Microalgae'; Published by BishenSingh.Mahend ra

Anand, N.; Handbook of Blue-Green Algae (of Rice Fields of South India)'; Publ. Bishen Singh Mahendra Pal Singh, Dehradun:1989

Bhoge, O. N. and G. Ragothaman; Studies on the Cyanophyceae from Jalgaon Region, Maharash tra'; Phykos 25:129-131:1986

Bhoge, O. N. and G. Ragothaman; Studies on the Euglenophyceae from Jalgaon Region, Maharash tra'; Phykos 25:132-135:1986

Desikachary, T. V.; 'Cyanophyta'; Indian Council of Agricultural Re search, New Delhi: 1-686:1959

Iyengar, M. O. P. and T. V. Desikachary; 'Volvocales'; Indian Council of Agrigultiral Research, New Delhi:1-532:1981

Lackey, J. B.; 'The manipulation andcounting of river plankton andchanges in some organisms due to formalin preservation'; U. S. Public Health Re ports:53:2080-2093:1938

Nandan, S. N. and N. H. Aher; 'Species diversity in algal flora of Haranbaree dam and Mosam river of Baglan (Maharashtra). J. Ecol. Envi. and Cons., $81(3-4):$ 551-553:2005

Pingle, S. D.; 'Studies on the algae of impoundments and streams in Maharashtra'; Ph. D. the sis, Poona University:198 1

Presscott, G. W.; 'How To Know the Fresh-Water Algae'; W. M. C Brown Company Publishers, Dubuque, IOWA:1954 